\title{
Lithium abundance and rotation of seismic solar analogues
}

\section{Solar and stellar connection from Kepler and HERMES observations ${ }^{\star}$}

\author{
P. G. Beck ${ }^{1}$, J.-D. do Nascimento Jr. ${ }^{2,3}$, T. Duarte ${ }^{2}$, D. Salabert ${ }^{1}$, A. Tkachenko ${ }^{4}$, S. Mathis ${ }^{1}$, \\ S. Mathur ${ }^{5}$, R. A. García ${ }^{1}$, M. Castro ${ }^{2}$, P. L. Pallé ${ }^{6,7}$, R. Egeland ${ }^{8,9}$, D. Montes ${ }^{10}$, O. Creevey ${ }^{11}$, \\ M. F. Andersen ${ }^{12}$, D. Kamath ${ }^{4}$, and H. van Winckel ${ }^{4}$
}

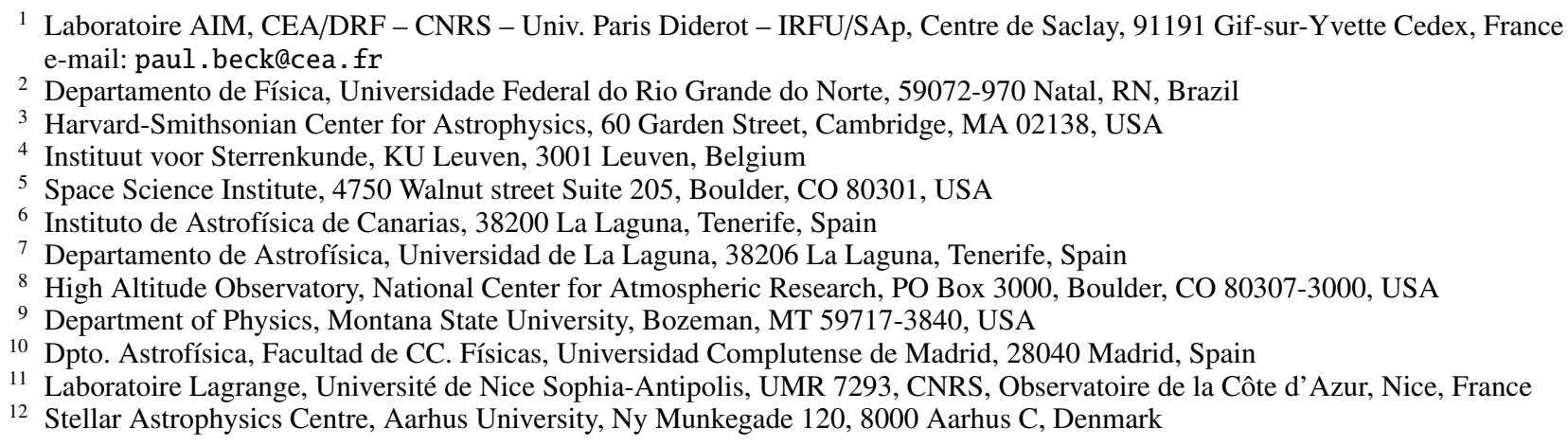

Received 30 September 2016 / Accepted 2 February 2017

\begin{abstract}
Context. Lithium abundance $A(\mathrm{Li})$ and surface rotation are good diagnostic tools to probe the internal mixing and angular momentum transfer in stars.

Aims. We explore the relation between surface rotation, $A(\mathrm{Li})$, and age in a sample of seismic solar-analogue stars, and we study their possible binary nature.

Methods. We selected a sample of 18 solar-analogue stars observed by the NASA Kepler satellite for an in-depth analysis. Their seismic properties and surface rotation rates are well constrained from previous studies. About $53 \mathrm{~h}$ of high-resolution spectroscopy were obtained to derive fundamental parameters from spectroscopy and $A(\mathrm{Li})$. These values were combined and compared with seismic masses, radii, and ages, as well as with surface rotation periods measured from Kepler photometry.

Results. Based on radial velocities, we identify and confirm a total of six binary star systems. For each star, a signal-to-noise ratio of $80 \lesssim S / N \lesssim 210$ was typically achieved in the final spectrum around the lithium line. We report fundamental parameters and $A(\mathrm{Li})$. Using the surface rotation period derived from Kepler photometry, we obtained a well-defined relation between $A(\mathrm{Li})$ and rotation. The seismic radius translates the surface rotation period into surface velocity. With models constrained by the characterisation of the individual mode frequencies for single stars, we identify a sequence of three solar analogues with similar mass $\left(\sim 1.1 M_{\odot}\right)$ and stellar ages ranging between 1 to $9 \mathrm{Gyr}$. Within the realistic estimate of $\sim 7 \%$ for the mass uncertainty, we find a good agreement between the measured $A(\mathrm{Li})$ and the predicted $A(\mathrm{Li})$ evolution from a grid of models calculated with the Toulouse-Geneva stellar evolution code, which includes rotational internal mixing, calibrated to reproduce solar chemical properties. We found a scatter in ages inferred from the global seismic parameters that is too large when compared with $A(\mathrm{Li})$.

Conclusions. We present the Li-abundance for a consistent spectroscopic survey of solar-analogue stars with a mass of $1.00 \pm 0.15 M_{\odot}$ that are characterised through asteroseismology and surface rotation rates based on Kepler observations. The correlation between $A(\mathrm{Li})$ and $P_{\text {rot }}$ supports the gyrochronological concept for stars younger than the Sun and becomes clearer when the confirmed binaries are excluded. The consensus between measured $A(\mathrm{Li})$ for solar analogues with model grids, calibrated on the Sun's chemical properties, suggests that these targets share the same internal physics. In this light, the solar Li and rotation rate appear to be normal for a star like the Sun.
\end{abstract}

Key words. stars: abundances - stars: fundamental parameters - stars: general - stars: interiors - asteroseismology

\section{Introduction}

In the past decade, numerous studies focused on the question whether the rotation and chemical abundances of the Sun are

\footnotetext{
^ Based on observations made with the NASA Kepler space telescope and the HERMES spectrograph mounted on the $1.2 \mathrm{~m}$ Mercator Telescope at the Spanish Observatorio del Roque de los Muchachos of the Instituto de Astrofísica de Canarias.
}

typical for a solar-type star, or in other words, for a star of solar mass and age (e.g. Gustafsson 1998; Allende Prieto et al. 2006; Delgado Mena et al. 2014; Datson et al. 2014; Ramírez et al. 2014; Carlos et al. 2016; dos Santos et al. 2016). These studies compared the Sun with solar-like stars and were inconclusive because of relatively large systematic errors (Gustafsson 2008; Robles et al. 2008; Reddy et al. 2003). 
The fragile element lithium is a distinguished tracer of mixing processes and loss of angular momentum inside a star (Talon \& Charbonnel 1998). Its abundance in stars changes considerably during the lifetime of the star. For low-mass stars on the main-sequence, proton-capture reactions destroy most of the initial stellar Li content in the stellar interior. Only a small fraction of the original $\mathrm{Li}$ is preserved in the cool outer convective envelopes. The solar photospheric $\mathrm{Li}$ abundance $A(\mathrm{Li})$ is $1.05 \pm 0.10 \mathrm{dex}$ (Asplund et al. 2009). This value is substantially lower than the protosolar nebular abundance of $A(\mathrm{Li})=3.3 \mathrm{dex}$ (Asplund et al. 2009) measured from meteorites, which illustrates that the lithium surface abundance does not reflect the original abundance of the star. A comparison between the Sun and typical stars of one solar mass and solar metallicity in the thin galactic disc by Lambert \& Reddy (2004) showed the Sun to be lithium-poor by a factor of 10 . Because the temperature at the base of the solar convective zone is not hot enough to destroy lithium, this large depletion in the observed solar to the meteoritic Li abundance by a factor of 160 remains one of the greatest challenges of standard solar models. This is known as the solar Li problem (e.g. Maeder 2009, Meléndez et al. 2010, and references therein).

There are two main challenges in understanding the Li abundance in stars similar to the Sun. First, for stars with masses $M \leq 1.1 M_{\odot}$, there is a strong dependence of $A(\mathrm{Li})$ on mass and metallicity, which are governed by the depth of the convective envelope for these stars (do Nascimento et al. 2009, 2010; Baumann et al. 2010; Castro et al. 2016). While the stellar chemical composition can be determined from optical spectroscopy, determining the mass of a star is a difficult task when a star does not belong to a cluster. In this context, solar analogues, following the classical definition of Cayrel de Strobel (1996), constitute a homogeneous set of stars in which mass and metallicity are well constrained with values close to solar as defined before. Fortunately, asteroseismology is a tool that provides precise and accurate values for mass, radius, and ages of oscillating stars (e.g. Aerts et al. 2010). The highest level of accuracy of the parameters determined through seismology is reached when the models are constrained by individual frequencies and combined with results from high-resolution spectroscopy (e.g. Chaplin et al. 2011, 2014; Lebreton \& Goupil 2014).

The second unknown is the complex interplay of various transport mechanisms and their efficiency inside the stellar interior and with stellar rotation. Standard models that only include mixing through convective motion fail to model the general trend of the $A(\mathrm{Li})$ evolution. This indicates that additional mixing processes have to be taken into account, such as microscopic diffusion (Eddington 1916; Chapman 1917), inertial gravity waves (GarciaLopez \& Spruit 1991; Schatzman 1996; Charbonnel \& Talon 2005), and the effects of stellar rotation. Rotation has a substantial effect on the stellar evolution (e.g. Zahn 1992; Maeder \& Zahn 1998; Brun et al. 1999; Mathis et al. 2004; Maeder 2009; Ekström et al. 2012, and references therein) and can change the properties of solar-type stars by reducing the effects of atomic diffusion and inducing extra mixing. More specifically, observations of light element abundances bring valuable constraints for mixing in models and transport processes in stars (Talon \& Charbonnel 1998; Charbonnel \& do Nascimento 1998; Pinsonneault 2010; Somers \& Pinsonneault 2016).

Numerous observational and theoretical studies have explored the Li surface abundance in the context of rotation, stellar evolution, age, and angular momentum transport (e.g. van den Heuvel \& Conti 1971; Skumanich 1972; Rebolo \& Beckman 1988; Zahn 1992; Zahn 1994; Charbonnel et al. 1994; Talon \& Charbonnel 1998; Talon \& Charbonnel 2005; Charbonnel \& do Nascimento 1998; King et al. 2000; Clarke et al. 2004; Pinsonneault 2010; Bouvier et al. 2016; Somers \& Pinsonneault 2016, and references therein). Recently, Bouvier et al. (2016) showed that a rotation-lithium relation exists even at an age of $5 \mathrm{Myr}$ and also exhibits a significant dispersion. Moreover, Bouvier (2008) proposed a possible link between lithium depletion and the rotational history of exoplanet-host stars. Thus, authors seek a complete and coherent description of the influence of rotation on the lithium abundances on the main sequence of solar-type stars. A particular challenge for studying $A(\mathrm{Li})$ as a function of the stellar rotation is the surface rotation velocity. If determined from spectroscopy through the Doppler broadening of the absorption lines, only the projected surface velocity $v \sin i$ could be measured, where the axis of the rotation axis remains unknown. When the surface rotation rate is determined through modulation of photometry or activity proxies, we measure the angular velocity in terms of surface rotation period, because a precise measure of the stellar radius is missing.

Understanding the evolution of the lithium abundance as a function of the mass, metallicity, and rotation and explaining its dispersion in $\mathrm{G}$ dwarfs is critical for constructing a comprehensive model of the Sun as a star (e.g. Pace et al. 2012; Castro et al. 2016). Comparing the measured value of $A(\mathrm{Li})$ in solar analogues with predictions from evolutionary models, calibrated to the solar case, will allow us to test the evolution of the Li dilution for typical "suns" at different ages. This gives us the possibility to test whether the mixing processes, assumed to act in the Sun, are peculiar or if the solar lithium value is normal.

This paper is structured as follows: in Sect. 2 we select the stars that are to be studied. We describe their properties and the new spectroscopic parameters. From the observations, the relation between lithium and rotation is discussed in Sect. 3. In Sect. 4 the measured $A(\mathrm{Li})$ is compared with theoretical predictions from the Toulouse-Geneva stellar evolution code (TGEC, Hui-Bon-Hoa 2008; do Nascimento et al. 2009), and we compare age estimates from seismology derived from previous studies using different approaches. The conclusions of this work are summarised in Sect. 5.

\section{Data set and stellar parameters}

The sample of solar analogues investigated in this study is composed of the 18 stars presented in Salabert et al. (2016a). A summary of the main global properties of these stars is provided in Table 1 . The stellar masses, radii, and ages reported in the literature (Table 1) were obtained by either grid-modelling analysis of the global seismic parameters or by using individual frequencies and high-resolution spectroscopy (hereafter also referred to as detailed modelling). For a specific discussion of the different modelling approaches, we refer to Lebreton \& Goupil (2014). Detailed modelling using individual frequencies with the Asteroseismic Modeling Portal (AMP, Metcalfe et al. 2009) is available for the following stars: KIC 3656476, KIC 4914923, KIC 6116048, KIC 7296438, KIC 7680114, KIC 9098294, and KIC 10644253, which were modelled by Mathur et al. (2012), Metcalfe et al. (2014), and Creevey et al. (2017). In this paper we use the latest results of Creevey et al. (2017) whenever possible. An additional star, KIC 3241581, has been modelled by Garcia et al. (in prep.; see also Beck et al. 2016), 
Table 1. Parameters found in the literature of the stars used in this study.

\begin{tabular}{lcccccc}
\hline \hline \multicolumn{1}{c}{ KIC } & $\begin{array}{c}v_{\max } \\
{[\mu \mathrm{Hz}]}\end{array}$ & $\begin{array}{c}M \\
{\left[M_{\odot}\right]}\end{array}$ & $\begin{array}{c}R \\
{\left[R_{\odot}\right]}\end{array}$ & $\begin{array}{c}P_{\text {rot }} \\
{[\text { days }]}\end{array}$ & $\begin{array}{c}\text { Age } \\
{[\mathrm{Gyr}]}\end{array}$ \\
\hline $3241581^{\star}$ & $2969 \pm 17$ & $1.04 \pm 0.02$ & $1.08 \pm 0.10$ & $26.3 \pm 2.0$ & $3.8 \pm 0.6$ & Ref. \\
$3656476^{\star}$ & $1947 \pm 78$ & $1.10 \pm 0.03$ & $1.32 \pm 0.01$ & $31.7 \pm 3.5$ & $8.9 \pm 0.4$ & 2 \\
$4914923^{\star}$ & $1844 \pm 73$ & $1.04 \pm 0.03$ & $1.34 \pm 0.02$ & $20.5 \pm 2.8$ & $7.0 \pm 0.5$ & 2 \\
5084157 & $1788 \pm 14$ & $1.06 \pm 0.13$ & $1.36 \pm 0.08$ & $22.2 \pm 2.8$ & $7.8 \pm 3.4$ & 3 \\
5774694 & $3671 \pm 20$ & $1.06 \pm 0.05$ & $1.00 \pm 0.03$ & $12.1 \pm 1.0$ & $1.9 \pm 1.8$ & 3 \\
$6116048^{\star}$ & $2098 \pm 84$ & $1.05 \pm 0.03$ & $1.23 \pm 0.01$ & $17.3 \pm 2.0$ & $6.1 \pm 0.5$ & 2 \\
6593461 & $2001 \pm 18$ & $0.94 \pm 0.16$ & $1.29 \pm 0.07$ & $25.7 \pm 3.0$ & $10.7 \pm 4.4$ & 3 \\
$7296438^{\star}$ & $1846 \pm 73$ & $1.10 \pm 0.02$ & $1.37 \pm 0.01$ & $25.2 \pm 2.8$ & $6.4 \pm 0.6$ & 2 \\
$7680114^{\star}$ & $1709 \pm 58$ & $1.09 \pm 0.03$ & $1.40 \pm 0.01$ & $26.3 \pm 1.9$ & $6.9 \pm 0.5$ & 2 \\
7700968 & $2010 \pm 25$ & $1.00 \pm 0.12$ & $1.21 \pm 0.06$ & $36.2 \pm 4.2$ & $7.5 \pm 3.1$ & 3 \\
9049593 & $1983 \pm 13$ & $1.13 \pm 0.14$ & $1.40 \pm 0.06$ & $12.4 \pm 2.5$ & $6.4 \pm 3.4$ & 3 \\
$9098294^{\star}$ & $2347 \pm 84$ & $0.98 \pm 0.02$ & $1.15 \pm 0.01$ & $19.8 \pm 1.3$ & $8.2 \pm 0.5$ & 2 \\
10130724 & $2555 \pm 27$ & $0.85 \pm 0.12$ & $1.08 \pm 0.05$ & $32.6 \pm 3.0$ & $13.8 \pm 5.0$ & 3 \\
10215584 & $2172 \pm 28$ & $0.99 \pm 0.13$ & $1.12 \pm 0.05$ & $22.2 \pm 2.9$ & $6.8 \pm 3.5$ & 3 \\
$10644253^{\star}$ & $2892 \pm 157$ & $1.09 \pm 0.09$ & $1.09 \pm 0.02$ & $10.9 \pm 0.9$ & $0.9 \pm 0.3$ & 2 \\
10971974 & $2231 \pm 6$ & $1.04 \pm 0.12$ & $1.09 \pm 0.03$ & $26.9 \pm 4.0$ & $5.8 \pm 3.0$ & 3 \\
11127479 & $1983 \pm 7$ & $1.14 \pm 0.12$ & $1.36 \pm 0.06$ & $17.6 \pm 1.8$ & $5.1 \pm 2.2$ & 3 \\
11971746 & $1967 \pm 23$ & $1.11 \pm 0.14$ & $1.35 \pm 0.06$ & $19.5 \pm 2.1$ & $6.0 \pm 2.8$ & 3 \\
\hline
\end{tabular}

Notes. Kepler input catalogue (KIC) number, central frequency of the oscillation power excess $v_{\max }$ by Chaplin et al. (2014), stellar mass and radius in solar units from seismology, surface rotation period $P_{\text {rot }}$ from García et al. (2014), stellar age from seismic modelling, and reference to the published seismic studies: [1] Garcia et al. (in prep., see also Beck et al. 2016); [2] Creevey et al. (2017) and [3] Chaplin et al. (2014). Stars whose parameters were obtained through the detailed modelling approach as described in Sect. 2 are flagged with an asterisk.

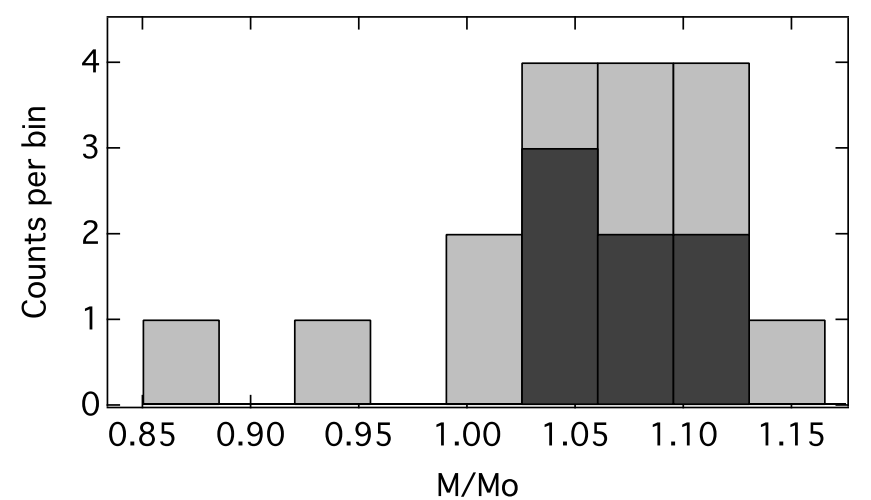

Fig. 1. Distributions of the stellar mass from seismology for the 18 solar-analogue stars in our sample. The grey bars flag the total distributions, while the dark shaded areas indicated the distribution of stars with detailed seismic modelling.

using the code Modules for Experiments in Stellar Astrophysics (MESA, Paxton et al. 2013, and references therein). For the remaining 10 stars, we adopted the masses and ages obtained by Chaplin et al. (2014) using global seismic parameters determined from one-month long Kepler time series and constraints on temperature and metallicity from multicolour photometry. The mass distribution in Fig. 1 shows that this sample mainly consists of stars with masses in the upper half of the allowed mass regime for solar analogues $\left(1-1.15 M_{\odot}\right)$. The dark shaded regions in Fig. 1 depict the distribution of the stars for which detailed seismic modelling was performed (Mathur et al. 2012; Metcalfe et al. 2014; Creevey et al. 2017; Garcia et al., in prep.). Whenever applicable, we distinguish in the diagrams represented in this paper the values originating from the two analysis approaches.

The surface rotation periods $\left(P_{\text {rot }}\right)$ measured by García et al. (2014) are reported in Table 1 . Selecting oscillating targets with known rotation periods adds the constraint that these stars are magnetically active. However, they are not so active as to suppress oscillations. This is another characteristic of our host star.

\subsection{Spectroscopic observations}

To guarantee a homogenous sample of fundamental parameters, we obtained for each target high-resolution spectra with the HERMES spectrograph (Raskin et al. 2011; Raskin 2011), which is mounted on the $1.2 \mathrm{~m}$ Mercator telescope in La Palma, Canary Island, Spain. The observations were performed in four observing runs of three to six days each. In 2015, spectroscopic data were obtained in June and July, while in 2016 the observations were made in April and May. The overview on the observations is presented in Table 2 . In total, $53.1 \mathrm{~h}$ worth of exposure time were collected. The HERMES spectra cover a wavelength range between 375 and $900 \mathrm{~nm}$ with a spectral resolution of $R \simeq 85000$. The wavelength reference was obtained from emission spectra of thorium-argon-neon reference frames in close proximity to the individual exposure.

The spectral reduction was performed with the instrumentspecific pipeline (Raskin et al. 2011; Raskin 2011). The radial velocity (RV) for each individual spectrum was determined from the cross correlation of the stellar spectrum in the wavelength range between 478 and $653 \mathrm{~nm}$ with a standardised G2-mask provided by the HERMES pipeline toolbox. For HERMES, the $3 \sigma$ level of the night-to-night stability for the observing mode described above is $\sim 300 \mathrm{~m} / \mathrm{s}$, which is used as the classical threshold for RV variations to detect binarity. Using the methods outlined by Beck et al. (2016), we corrected individual spectra for the Doppler shift before normalisation and the process of combining individual spectra. The signal-to-noise ratios $(\mathrm{S} / \mathrm{N})$ of each combined spectrum around $670 \mathrm{~nm}$ are reported in Table 3 and in Fig. 2. A solar flux spectrum was observed with the same HERMES instrument setup in reflected sunlight from the 
Table 2. Summary of the seismic solar analogue observations.

\begin{tabular}{|c|c|c|c|c|c|c|c|}
\hline KIC & $\begin{array}{c}V \\
{[\mathrm{mag}]}\end{array}$ & $N$ & $\begin{array}{l}\text { ToT } \\
{[\mathrm{hrs}]}\end{array}$ & $\begin{array}{c}\Delta T \\
{[\text { days] }}\end{array}$ & $\begin{array}{c}\overline{\mathrm{RV}} \\
{\left[\mathrm{km} \mathrm{s}^{-1}\right]}\end{array}$ & $\begin{array}{c}\Delta \mathrm{RV} \\
{\left[\mathrm{km} \mathrm{s}^{-1}\right]}\end{array}$ & Comment \\
\hline $3241581^{\star}$ & $10.35 \pm 0.04$ & 24 & 9.3 & 709.1 & -30.68 & 0.96 & binary \\
\hline 3656476 & $9.55 \pm 0.02$ & 6 & 2.5 & 351.1 & -13.23 & 0.14 & \\
\hline 4914923 & $9.50 \pm 0.02$ & 7 & 2.1 & 297.3 & -31.16 & 2.11 & binary \\
\hline 5084157 & $11.56 \pm 0.12$ & 10 & 5.2 & 300.1 & -19.66 & 0.21 & \\
\hline 5774694 & $8.37 \pm 0.01$ & 7 & 1.3 & 348.1 & -17.67 & 0.16 & \\
\hline 6116048 & $8.47 \pm 0.01$ & 5 & 1.0 & 347.2 & -53.28 & 0.17 & \\
\hline 6593461 & $11.22 \pm 0.10$ & 9 & 4.1 & 296.2 & -35.39 & [0.37] & large scatter \\
\hline 7296438 & $10.13 \pm 0.03$ & $(+2) 4$ & 1.5 & 350.2 & -2.08 & 16.65 & binary (KOI 364.01) \\
\hline 7680114 & $10.15 \pm 0.04$ & 8 & 3.4 & 351.0 & -58.96 & 0.180 & \\
\hline 7700968 & $10.37 \pm 0.04$ & $(+2) 4$ & 1.4 & 299.1 & +39.47 & 27.62 & binary \\
\hline 9049593 & $10.35 \pm 0.04$ & 4 & 1.5 & 299.1 & -21.02 & 0.24 & \\
\hline 9098294 & $9.91 \pm 0.03$ & 7 & 2.6 & 346.1 & -55.78 & 41.35 & binary \\
\hline 10130724 & $12.03 \pm 0.19$ & 7 & 2.8 & 299.1 & -54.51 & 2.12 & binary \\
\hline 10215584 & $10.62 \pm 0.05$ & 6 & 2.7 & 337.0 & -11.02 & 0.25 & \\
\hline 10644253 & $9.26 \pm 0.02$ & 14 & 5.9 & 416.0 & -19.01 & 0.18 & \\
\hline 10971974 & $11.05 \pm 0.07$ & 4 & 1.6 & 300.1 & -34.58 & 0.27 & \\
\hline 11127479 & $11.21 \pm 0.09$ & 5 & 2.1 & 300.2 & -29.33 & 0.30 & KOI 2792.01, large scatter \\
\hline 11971746 & $11.00 \pm 0.07$ & 8 & 3.6 & 301.0 & -44.20 & 0.22 & \\
\hline
\end{tabular}

Notes. The star identifier in the Kepler input catalogue (KIC), apparent magnitude in Johnson V, number $N$ of spectra (in brackets the number of additional spectra with $\mathrm{S} / \mathrm{N}$ only high enough to determine the radial velocity), total accumulated time on target (ToT), the time base $\Delta T$ covered by the observations, the mean radial velocity $(\overline{\mathrm{RV}})$, and the difference between the positive and negative extrema of the measured RV values and their internal error, and a comment on the star/system. KOI stands for Kepler Objects of Interests and indicates planet host star candidates.

Table 3. Fundamental parameters of the solar analogues from the spectroscopic analysis of HERMES data.

\begin{tabular}{rlcccrrr}
\hline \hline KIC & \multicolumn{1}{c}{$\begin{array}{c}T_{\text {eff }} \\
{[\mathrm{K}]}\end{array}$} & $\begin{array}{c}\log g \\
{[\mathrm{dex}]}\end{array}$ & $\begin{array}{c}v_{\min } \\
{\left[\mathrm{km} \mathrm{s}^{-1}\right]}\end{array}$ & $\begin{array}{c}v \sin i \\
{\left[\mathrm{~km} \mathrm{~s}^{-1}\right]}\end{array}$ & $\begin{array}{c}{[\mathrm{Fe} / \mathrm{H}]} \\
{[\mathrm{dex}]}\end{array}$ & $\begin{array}{r}A(\mathrm{Li}) \\
{[\mathrm{dex}]}\end{array}$ & $\begin{array}{r}S / N \\
(\mathrm{Li})\end{array}$ \\
\hline 3241581 & $5685 \pm 59$ & $4.3 \pm 0.1$ & $1.0 \pm 0.2$ & $4.0 \pm 0.6$ & $0.22 \pm 0.04$ & $\leq 0.31$ & 180 \\
3656476 & $5674 \pm 50$ & $4.2 \pm 0.1$ & $1.1 \pm 0.1$ & $4.1 \pm 0.7$ & $0.25 \pm 0.04$ & $\leq 0.51$ & 120 \\
4914923 & $5869 \pm 74$ & $4.2 \pm 0.1$ & $1.2 \pm 0.1$ & $5.0 \pm 0.6$ & $0.12 \pm 0.04$ & $2.02 \pm 0.02$ & 175 \\
5084157 & $5907 \pm 60$ & $4.2 \pm 0.1$ & $1.1 \pm 0.1$ & $4.8 \pm 0.7$ & $0.24 \pm 0.04$ & $2.31 \pm 0.01$ & 85 \\
5774694 & $5962 \pm 59$ & $4.6 \pm 0.1$ & $1.0 \pm 0.2$ & $5.5 \pm 0.7$ & $0.10 \pm 0.03$ & $2.60 \pm 0.01$ & 190 \\
6116048 & $6129 \pm 97$ & $4.3 \pm 0.2$ & $1.3 \pm 0.2$ & $5.8 \pm 0.6$ & $-0.18 \pm 0.05$ & $2.61 \pm 0.01$ & 150 \\
6593461 & $5803 \pm 126$ & $4.4 \pm 0.2$ & $1.3 \pm 0.3$ & $4.8 \pm 0.8$ & $0.25 \pm 0.09$ & $1.85 \pm 0.02$ & 70 \\
7296438 & $5854 \pm 64$ & $4.3 \pm 0.1$ & $1.2 \pm 0.2$ & $4.5 \pm 0.8$ & $0.24 \pm 0.05$ & $1.89 \pm 0.03$ & 80 \\
7680114 & $5978 \pm 107$ & $4.3 \pm 0.2$ & $1.2 \pm 0.3$ & $4.4 \pm 0.7$ & $0.15 \pm 0.07$ & $1.57 \pm 0.02$ & 135 \\
7700968 & $5992 \pm 144$ & $4.4 \pm 0.3$ & $1.4 \pm 0.4$ & $5.3 \pm 0.7$ & $-0.18 \pm 0.08$ & $2.19 \pm 0.02$ & 100 \\
9049593 & $6009 \pm 151$ & $4.3 \pm 0.3$ & $1.5 \pm 0.3$ & $7.2 \pm 0.6$ & $0.20 \pm 0.08$ & $2.82 \pm 0.02$ & 95 \\
9098294 & $5913 \pm 67$ & $4.4 \pm 0.1$ & $1.0 \pm 0.2$ & $4.7 \pm 0.7$ & $-0.14 \pm 0.04$ & $\leq 0.86$ & 140 \\
10130724 & $5649 \pm 95$ & $4.3 \pm 0.2$ & $0.9 \pm 0.3$ & $4.4 \pm 0.9$ & $0.27 \pm 0.09$ & $\leq 1.10$ & 65 \\
10215584 & $5888 \pm 67$ & $4.3 \pm 0.1$ & $1.1 \pm 0.2$ & $5.1 \pm 0.6$ & $0.05 \pm 0.04$ & $2.25 \pm 0.05$ & 105 \\
10644253 & $6117 \pm 64$ & $4.4 \pm 0.2$ & $0.9 \pm 0.2$ & $4.3 \pm 0.6$ & $0.11 \pm 0.04$ & $2.99 \pm 0.02$ & 215 \\
10971974 & $5895 \pm 114$ & $4.4 \pm 0.2$ & $1.2 \pm 0.3$ & $4.8 \pm 0.8$ & $0.02 \pm 0.07$ & $\leq 1.62$ & 55 \\
11127479 & $5884 \pm 116$ & $4.4 \pm 0.3$ & $1.5 \pm 0.3$ & $6.1 \pm 0.7$ & $0.11 \pm 0.08$ & $2.44 \pm 0.02$ & 50 \\
11971746 & $5953 \pm 63$ & $4.3 \pm 0.1$ & $1.2 \pm 0.2$ & $4.5 \pm 0.8$ & $0.18 \pm 0.04$ & $2.19 \pm 0.02$ & 105 \\
\hline
\end{tabular}

Notes. The star identifier in the Kepler input catalogue, the effective temperature $T_{\text {eff }}$, the surface acceleration $\log g$, the micro turbulence $v_{\text {min }}$, the projected surface rotational velocity $v \sin i$, the stellar metallicity, and the abundance of lithium are given with their respective uncertainties. Upper limits of the measured $A(\mathrm{Li})$ are indicated for stars with low lithium abundance as a consequence of insufficient $\mathrm{S} / \mathrm{N}$ in the spectra. The last column reports the $\mathrm{S} / \mathrm{N}$ around the lithium line.

Jovian moon Europa (Beck et al. 2016). This spectrum has a $S / N \sim 450$ (Fig. 2).

\subsection{Fundamental parameters}

To determine the fundamental parameters, we started with the effective temperature $T_{\text {eff }}$, the surface gravity $\log g$, the metallicity
$[\mathrm{Fe} / \mathrm{H}]$, and the microturbulence $v_{\min }$ and projected surface rotational velocity $v \sin i$ from an analysis with the Grid Search in Stellar Parameters $\left(\right.$ GSSP $\left.^{1}\right)$ software package (Lehmann et al. 2011; Tkachenko et al. 2012; Tkachenko 2015). The library of

\footnotetext{
1 The GSSP package is available for download at https: //fys.kuleuven.be/ster/meetings/binary-2015/ gssp-software-package
} 


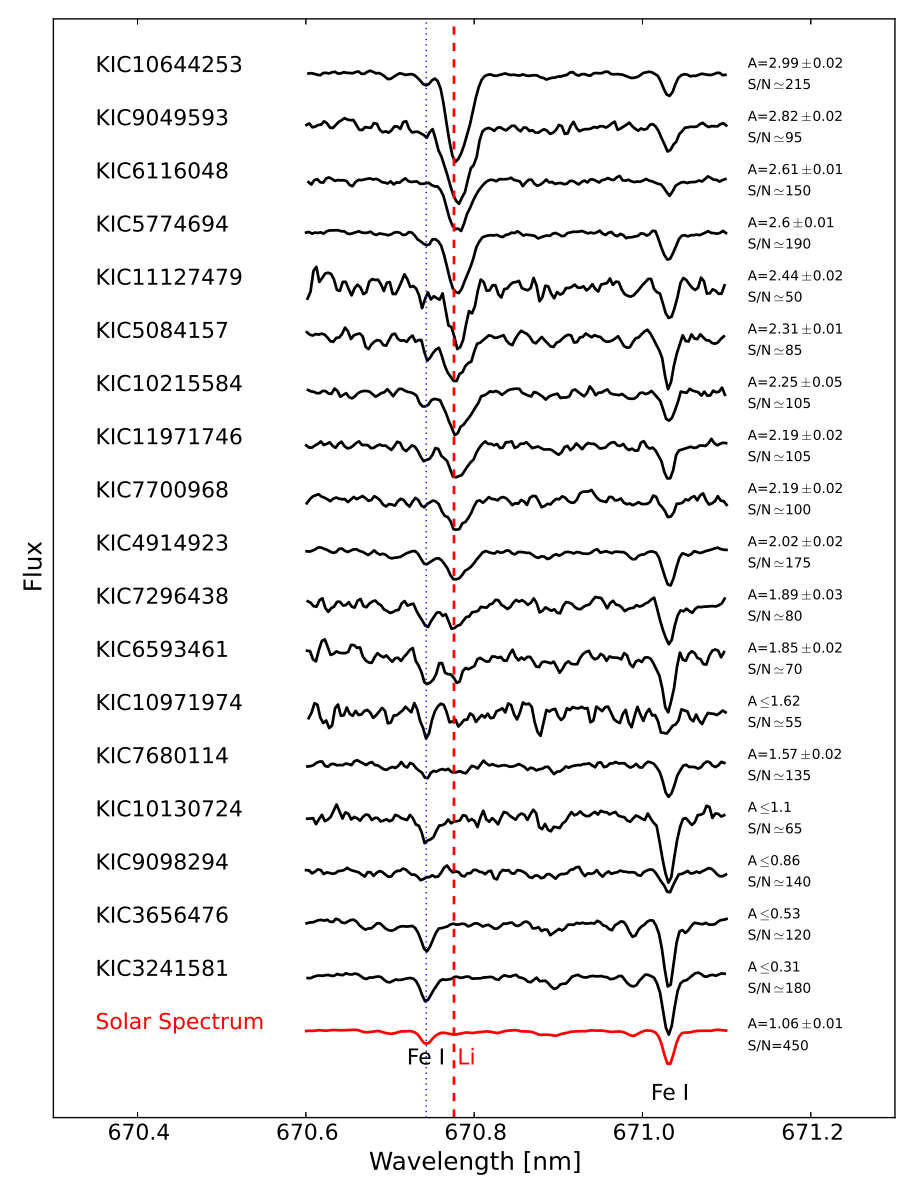

Fig. 2. Lithium doublet observed in the full data set, sorted from strong to weak lithium lines (top to bottom). The solar spectrum (red) is shown in the bottom of the diagram. The centre of the lithium and the neighbouring iron line are plotted as the vertical dashed line and blue dotted line, respectively. The achieved $\mathrm{S} / \mathrm{N}$, measured lithium abundances, and upper limits of $A(\mathrm{Li})$ are indicated at the right side.

synthetic spectra was computed using the SYNTHV radiative transfer code (Tsymbal 1996), which is based on the LLMODELS code (Shulyak et al. 2004). Then, we used the HERMES highquality spectra to determine the final stellar fundamental parameters $\left(T_{\text {eff }}, \log g,[\mathrm{Fe} / \mathrm{H}]\right)$ and lithium abundance. We employed the excitation/ionisation equilibrium balance technique to find the stellar parameters that produced consistent abundances of Fe I and Fe II, and by using the solar reference value as described by Meléndez et al. (2012) and Ramírez et al. (2014). For all stars in the sample we determined the fundamental stellar parameters by performing a differential excitation-ionisation equilibrium from the abundances of Fe I and Fe II, and by using the solar value as a reference, as described by Meléndez et al. (2012, 2014b), Monroe et al. (2013), and Ramírez et al. (2014). We combined Kurucz atmospheric models (Castelli \& Kurucz 2004) with equivalent width (EW) measurements of Fe I and Fe II and the 2014 version of the 1D LTE code MOOG (Sneden 1973). The EW were determined from the automated code ARES (Sousa et al. 2007). We applied the same method for all stars in our sample, considering the same regions of continuum. Final spectroscopic parameters for the stars are given in Table 3. Formal uncertainties of the stellar parameters were computed as in Epstein et al. (2010) and Bensby et al. (2014). The median metallicity for the sample is 0.15 dex. We note that we find a higher temperature for KIC 10644253 than the previous findings

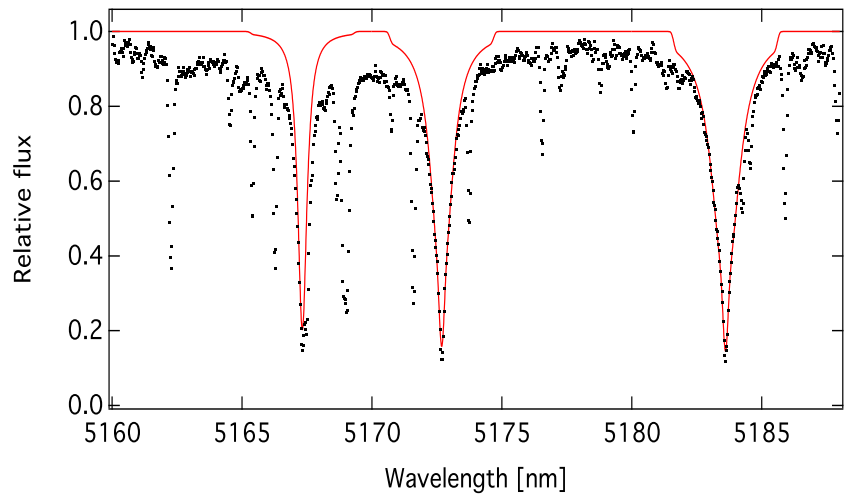

Fig. 3. Mg triplet in the star KIC 7700968. The observed and synthetic spectra are represented by black dots and the solid line, respectively.

of Salabert et al. (2016b). We adopt this new value because the analysis was improved through increased observing time and by applying a differential analysis with the HERMES solar spectrum. We adopt the values listed in Table 3. For KIC 3241581, we confirm the results reported previously by Beck et al. (2016).

The wings of Balmer and Mg-lines in cool dwarfs stars are highly sensitive to the temperature, $\log g$, and metallicity (Gehren 1981; Fuhrmann et al. 1993; Barklem \& O'Mara 2001). These lines are formed in deep layers of the stellar atmosphere, and they are expected to be insensitive to the non-LTE effects (Barklem 2007), although they depend on convection (Ludwig et al. 2009). The comparison between the observed and synthetic spectra for the region between 516.0 and $518.8 \mathrm{~nm}$, containing the Mg-triplet and a sufficient number of metal lines, shown in Fig. 3. The agreement of the line widths shows the quality of our determined fundamental parameters $\left(T_{\mathrm{eff}}, \log g\right.$, $[\mathrm{Fe} / \mathrm{H}])$.

\subsection{Lithium abundance}

The $A(\mathrm{Li})$ was derived from the $\mathrm{Li}$ I resonance doublet feature at $670.78 \mathrm{~nm}$ as depicted for all stars in our sample in Fig. 2. We used the "synth" driver of the 2014 version code MOOG (Sneden 1973) and adopted $A(\mathrm{Li})_{\odot}=1.05 \mathrm{dex}$ as the standard solar lithium abundance (Asplund et al. 2009). The atmosphere models we used were interpolated from the new Kurucz grid (Castelli \& Kurucz 2004) for a set of spectroscopic atmospheric parameters, $T_{\text {eff }}, \log g$, $[\mathrm{Fe} / \mathrm{H}]$, and microturbulence as given in Table 3. We used the Fe I and Fe II absorption lines as specified in Meléndez et al. (2014a), and we neglected possible ${ }^{6} \mathrm{Li}$ influences. Owing to the vicinity of the $\mathrm{Li}$ lines to the Fe I line at $670.78 \mathrm{~nm}$ (blue dotted line in Fig. 2), strong Li or iron lines as well as fast rotation can lead to blended lines. Therefore, an accurate value of the iron abundance, the $\log g$, and the projected surface velocity is needed to correctly derive the lithium abundance. The main sources for the Li abundance error are related to the uncertainties on the stellar parameters and the EW measurement. However, $T_{\mathrm{eff}}$ is by far the dominant source of error. For the spectroscopic atmospheric parameters ${ }^{2}$, we determined the lithium abundance in our sample, which ranges between 0.06 and 3.03 dex. For comparison, the solar lithium abundance was also derived from the HERMES solar spectrum (Fig. 2) collected from the reflected light of the Jovian moon

\footnotetext{
2 In this work, we use the standard definitions $[\mathrm{X} / \mathrm{Y}]=\log \left(\mathrm{N}_{\mathrm{X}} / \mathrm{N}_{\mathrm{Y}}\right)-$ $\log \left(\mathrm{N}_{\mathrm{X}} / \mathrm{N}_{\mathrm{Y}}\right)_{\odot}$, and $A_{\mathrm{X}}=\log \left(\mathrm{N}_{\mathrm{X}} / \mathrm{N}_{\mathrm{H}}\right)+12$, where $N_{\mathrm{X}}$ is the number density of element $\mathrm{X}$ in the stellar photosphere.
} 


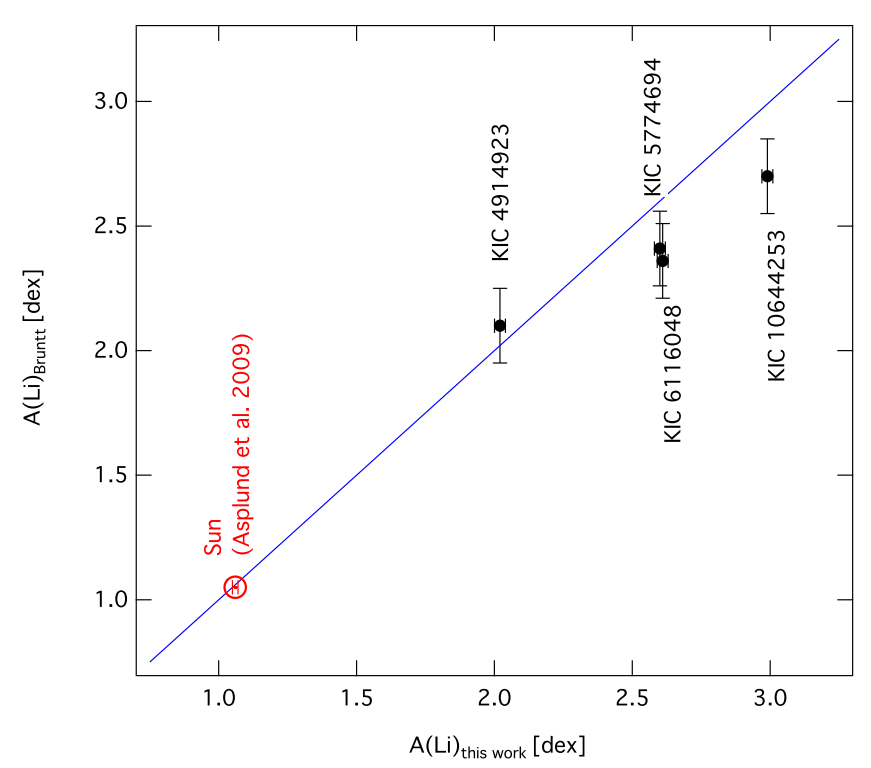

Fig. 4. Comparison of the measured lithium abundances with values from the literature (see Table 4). Black squares show the four stars from our sample that overlap with the stars of Bruntt et al. (2012). The red circle depicts the comparison of the solar lithium abundance derived from our spectrum with the canonical value by Asplund et al. (2009). The blue line denotes the 1:1 ratio between the two data sets.

Table 4. Comparison of lithium abundances with other values in the literature.

\begin{tabular}{rrrc}
\hline \hline \multicolumn{1}{c}{ Star } & \multicolumn{2}{c}{$A(\mathrm{Li})[\mathrm{dex}]$} & Literature \\
& This work & Literature & reference \\
\hline KIC 4914923 & $2.02 \pm 0.02$ & $2.1 \pm 0.2$ & $\mathrm{~B} 12$ \\
KIC 5774694 & $2.6 \pm 0.01$ & $2.4 \pm 0.2$ & $\mathrm{~B} 12$ \\
KIC 6116048 & $2.61 \pm 0.01$ & $2.4 \pm 0.2$ & $\mathrm{~B} 12$ \\
KIC 10644253 & $2.99 \pm 0.02$ & $2.7 \pm 0.2$ & $\mathrm{~B} 12$ \\
\hline Sun & $1.06 \pm 0.01$ & $1.05 \pm 0.1$ & $\mathrm{~A} 09$ \\
\hline
\end{tabular}

Notes. The stellar identifier, the lithium abundance derived in this work, and the literature value provided by Bruntt et al. (2012; B12) or Asplund et al. (2009; A09). The comparison is depicted in Fig. 4.

Europa. We measured $A(\mathrm{Li})_{\odot}=1.06 \pm 0.1 \mathrm{dex}$ in agreement with Asplund et al. (2009). The final values of $A(\mathrm{Li})$ are listed in the last column of Table 3 and Fig. 2. Figure 2 also illustrates the sequence of spectral segments, which contains the two lithium as well as two iron lines for all stars in our sample, sorted by decreasing value of $A(\mathrm{Li})$. For comparison, the solar spectrum obtained by Beck et al. (2016) is plotted at the bottom of the sequence. The comparison between our derived abundances with the values from Bruntt et al. (2012) is presented in Fig. 4. A good agreement was found between the values for $A(\mathrm{Li})$. The differences probably originate from the NLTE effects in the hotter stars (Lind et al. 2009).

\subsection{Binarity occurrence}

The time span covered by our measurements together with the mean value and the dispersion of the radial velocities are reported in Table 3 for each star in the sample. The measurements range between 260 and 700 days. Based on an earlier analysis of KIC 3241581, Beck et al. (2016) confirmed this star to be a binary with an orbital period longer than $1.5 \mathrm{yr}$. Based on the first 35 days of the observations of this campaign, Salabert et al. (2016a) reported KIC 4914923, KIC 7296438, and KIC 9098294 as binary candidates. Additional spectra were needed to confirm the binarity status of these systems. From the full available data set we analysed in this paper, we confirm that the three systems are binaries, and we report that KIC 10130724 and KIC 7700968 are also binary systems.

For none of the systems a binary period is known as yet because we do not detect a signature of stellar binarity (eclipses or tidally induced flux modulation) in their light curves. In addition, the RV measurements are too sparsely sampled to derive an orbital period from it. Therefore no meaningful upper or even lower limit can be proposed on the orbital periods. The mean value reported in Table 2 will roughly resemble the systemic velocity of the binary system. Without information on the orbital parameters, the interpretation of $A(\mathrm{Li})$ in the stellar components of the system is not reliable. Therefore, continuous RV monitoring is required to draw further constraints on the orbital parameters, such as period or eccentricity.

\section{Lithium abundance and surface rotation}

Many observational works have studyied the connection between $\mathrm{Li}$ and rotation in a search for correlations between these parameters (e.g. Skumanich 1972; Rebolo \& Beckman 1988; King et al. 2000; Clarke et al. 2004; Bouvier 2008; Bouvier et al. 2016). Because of the difficulty in coverage and stability of photometric follow-up observations, most of them have employed $v \sin i$ measurements. Owing to the undetermined inclination angle $i$, these values yield a lower limit on the rotational velocity. The rotation period from the light-curve modulation, such as determined by Krishnamurthi et al. (1998) for the Pleiades or from the modulation of the emission in the core of the Ca H\&K lines (e.g. Choi et al. 1995), is independent of the inclination. This, linked to the observational difficulty of resolving the tiny absorption line of lithium at $670.7 \mathrm{~nm}$ with high $\mathrm{S} / \mathrm{N}$ for the solar-analogue stars, partially explains the difficulty of connecting the dependence between true rotation (rotational period) and the lithium abundance in low-mass solar-analogues stars at different ages.

In the left panel of Fig. 5, the lithium abundance is plotted as a function of the surface rotation period derived from the Kepler light curves (García et al. 2014) and surface rotation velocity. For comparison, the Sun is presented by the longitudinal average rotation period of 27 days. The full range of solar differential rotation is spreads between 25 days at the equator and 34 days at the poles (e.g. Thompson et al. 1996), and it is represented by the horizontal error bar in the solar symbol in Fig. 5. This figure shows that fast-rotating stars have high Li abundances. This confirms the well-known general trend for lithium and rotation found in the earlier mentioned studies of clusters and for single field stars (e.g. Skumanich 1972; Rebolo \& Beckman 1988; King et al. 2000; Clarke et al. 2004; Bouvier 2008; Bouvier et al. 2016). There is large scatter for rotation periods longer than the solar rotation period. This is also found in similar studies of clusters and large samples of field stars. In this context, our sample is unique in the sense that we combine for field stars the existing information about the true rotation period, lithium abundance, seismic age and masses, and binarity status. In addition, stellar binarity can affect the measured lithium abundance either through interactions of the components (e.g. Zahn 1994) or through observational biases. Because an orbital solution is needed for a complete analysis of 

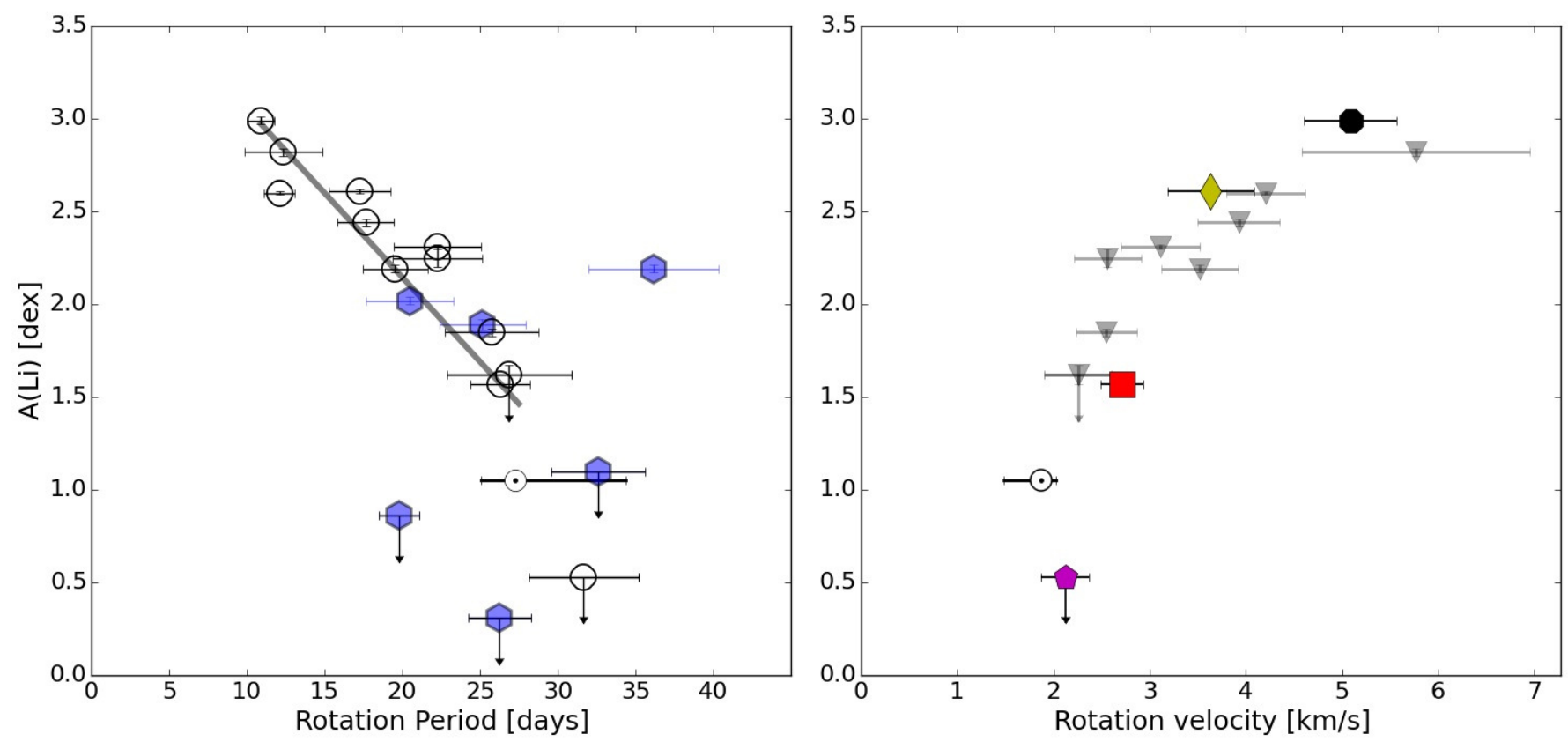

Fig. 5. Lithium abundance versus rotation for the 18 seismic solar analogues. The left and right panels compare $A(\mathrm{Li})$ with the surface rotation period from space photometry and the computed surface rotation velocity, respectively. Stars found to be located in binaries are shown as filled blue octagons in the left-hand panel, and they are removed from the right panel. In both panels, the full range covered by the differential solar rotation is represented by the horizontal error bar in the solar symbol. The solid line in the left panel depicts the best-fitting relation for single stars between the rotation period and $A(\mathrm{Li})$ for stars with rotation periods shorter than the solar value. In the right panel, the source of the asteroseismic radius is illustrated through the choice of symbols. Triangle markers indicate stars whose radius has been determined through global parameter modelling. Upper limits of the measured $A(\mathrm{Li})$ are shown for stars with low lithium abundance or insufficient $\mathrm{S} / \mathrm{N}$ in the spectra. The single stars with the radius from detailed modelling from AMP, KIC 10644253, KIC 6116048, KIC 7680114, and KIC 3656476, are plotted as black octagon, yellow diamond, red square, and magenta pentagon, respectively.

such system, we concentrate on the single stars in this work. This approach reduces the scatter in the $A(\mathrm{Li})-P_{\text {rot }}$ plane when only single stars are taken into account. In the remaining analysis we only use single stars and stars with $P_{\text {rot }}$ shorter than 27 days. For this subsample, the Li-rotation correlation shows a trend following a linear regression,

$A(\mathrm{Li})=-(0.08 \pm 0.01) \times P_{\text {rot }}+(3.85 \pm 0.17)$.

This relation indicates that lithium appears to evolve similarly to the rotation velocity for stars in this range of mass and metallicities for $P_{\text {rot }} \lesssim 27$ days. We note that by fitting a trend in the $P_{\text {rot }}$-Li plane, we do not explicitly take the age of the stars into account. It is taken into account implicitly, however, because surface rotation is a proxy of age (Barnes 2007), although van Saders et al. (2016) showed that when stars reach approximately the age of the Sun, they do not slow down as much as predicted by the empirical relations between rotation and stellar age (e.g. Barnes 2007; Gallet \& Bouvier 2013, 2015). However, gyrochronology is still valid for stars younger than the Sun. A rotation rate higher than the solar value therefore implies a star younger than the Sun. This is in agreement with the modelled evolution of Li because the strongest depletion of the lithium surface abundance on the main sequence occurs in the early stages (e.g. Castro et al. 2016, and references therein; see also Sect. 5 in this paper).

The evolution of the lithium abundance with rotation period as described by Eq. (1) is the best fit of the trend for stars with masses $1.0 \lesssim M / M_{\odot} \lesssim 1.1$ and $[\mathrm{Fe} / \mathrm{H}]$ in the range 0.1 to 0.3 dex (with the exceptions of KIC 10971974 and KIC 6116048, Table 3). The trend is well defined for rotation periods shorter than 27 days. The bulk of stars in our sample rotates with rotation periods shorter than the period of the Sun. This could be due to a selection bias in which longer periods are more difficult to detect. In addition, more data seem to be necessary to extrapolate these results for slow-rotator (long-period) regimes.

For two stars with the same angular velocity (as used in the left panel of Fig. 5), the rotational velocity still can be different, however, since it depends on the unknown stellar radius. We can overcome this degeneracy by using the asteroseismic radius (Table 1) to convert the rotation period into the unprojected rotational velocity $v_{\text {rot }}$ in kilometers-per-second. In the right panel of Fig. 5, we plot the rotational velocity computed as a function of lithium abundance. Because spots can be found at a relatively wide range of latitudes, surface differential rotation might contribute to the scatter in the rotation-lithium relation. Furthermore, the flux modulation introduced by spots, in combination with an assumed solar or anti-solar differential rotation profile, will lead to an under- or over-estimation of the rotational velocity at the equator, respectively (Brun et al. 2015; Brun \& Browning 2017). From the right panel of Fig. 5 we find that for stars with rotational velocities higher than $3 \mathrm{~km} \mathrm{~s}^{-1}$ a linear trend is found in the Li-velocity relation. Between values of $v_{\text {rot }}$ of 2 and $3 \mathrm{~km} \mathrm{~s}^{-1}$, a large dispersion in the measured values of lithium could be present $(A(\mathrm{Li}) \lesssim 2 \mathrm{dex})$ that also engulfs the position of the Sun in this diagram. When we compare the right panel of Fig. 5 to large-sample studies of $v \sin i$, such as Takeda et al. (2010), the agreement is good. In the asteroseismic approach, the complications introduced by the unknown inclination of the rotation axis or the assumptions on the turbulent velocity fields that could influence the line profile are eliminated. Applying this asteroseismic approach on large samples should thus reduce the systematic scatter. 


\section{Discussion}

The two observables, rotation and $A(\mathrm{Li})$, are expected to evolve with time for stars of this mass range during the main sequence. This was suggested by Skumanich (1972) from the observations of stars in the Hyades, Pleiades, Ursa Major, and the Sun, which were further investigated (e.g. Rebolo \& Beckman 1988; King et al. 2000; Clarke et al. 2004). In general, the $\mathrm{Li}$ abundance is a function of the convective envelope deepening relative to the age of a star on the main sequence (do Nascimento et al. 2009; Baumann et al. 2010). It can also be the consequence of mixing below the convective zone (Brun et al. 1999) or in the radiative core (Talon \& Charbonnel 2005; Charbonnel \& Talon 2005). This confirms that the age, the angular momentum history, the mass, and the metallicity are the governing physical processes in the evolution of the lithium content. Recently, Castro et al. (2016) showed that the cluster M67 exhibits a relatively large scatter of the lithium abundance in the main-sequence stars with the same effective temperature and same age. The scatter is the largest around the $1 M_{\odot}$ range $(0.5 \lesssim A(\mathrm{Li}) \lesssim 2.0 \mathrm{dex})$ and suggests that another as yet unknown process could influence the lithium abundance. Somers \& Pinsonneault (2016) suggested that an intrinsically different mixing history than in other stellar clusters, such as a higher fraction of fast rotators or inhomogeneities of the initial rotation distribution (Coker et al. 2016), could explain the scatter in lithium depletion for stars older than $100 \mathrm{Myr}$.

\subsection{Stellar ages}

In a cluster all stars have the same age. This cannot be assumed for the field stars in our sample. As described in Sect. 2, there are two ways to use the seismic information to infer the age. When grid-modelling based on global seismic parameters is used, the inferred ages have large uncertainties (Lebreton \& Goupil 2014). In our sample, several of these stars are rotating faster than the Sun (García et al. 2014), which indicates that they are probably younger (Barnes 2007; van Saders et al. 2016). However, some ages are derived from global-parameter seismology, and are older than the solar age. To avoid these inconsistency problems, in the following analysis we only use ages inferred from detailed modelling that are constrained by individual frequencies or frequency ratios, as described in Sect. 2 and listed in Table 1. We have age and mass estimates through this approach for eight stars from studies of Mathur et al. (2012), Metcalfe et al. (2014), Creevey et al. (2017), and Garcia et al. (in prep.).

\subsection{Comparison with stellar models}

To compare the derived stellar age and measured lithium abundance with stellar modelling predictions, we computed a grid of models of the temporal evolution of $A(\mathrm{Li})$ that is due to rotation-induced mixing using the Toulouse-Geneva stellar evolution code (TGEC, Hui-Bon-Hoa 2008; do Nascimento et al. 2009).

A description of the physics used for this grid is given in Appendix A. For details on the calculation of the theoretical $\mathrm{Li}$ abundance we refer to Castro et al. $(2009,2016)$ and do Nascimento et al. (2009). These models include the effect of the rotation-induced mixing on chemicals due to the combined actions of meridional circulation and shear-induced turbulence in stellar radiation zones computed as prescribed by Zahn (1992), Maeder \& Zahn (1998), Théado \& Vauclair (2003), and

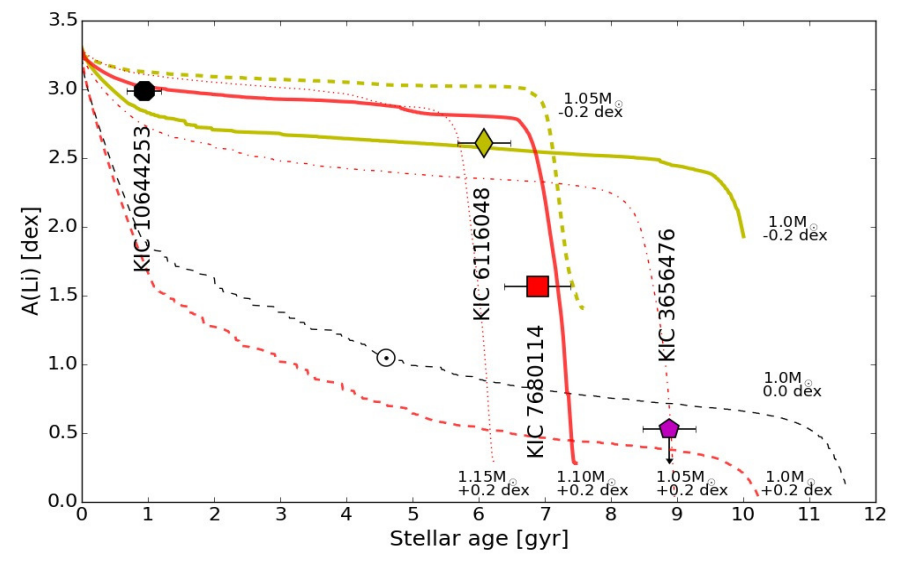

Fig. 6. Lithium abundance of single stars with age computed by detailed modelling with the AMP code. The stars KIC 10644253, KIC 6116048, KIC 7680114, and KIC 3656476 are plotted as black hexagon, yellow diamond, red square, and magenta pentagon, respectively. For KIC 3656476 the upper limits of the measured $A(\mathrm{Li})$ is shown. TGECevolutionary tracks are shown in red, and yellow represents the theoretical evolution of $A(\mathrm{Li})$ for models of the indicated mass with $[\mathrm{Fe} / \mathrm{H}]=+0.2$, and -0.2 dex, respectively. The black dashed evolutionary track depicts the evolution of Li calculated by do Nascimento et al. (2009), and the solar marker depicts the measured $\mathrm{A}\left(\mathrm{Li}_{\odot}\right)$ and age of the Sun.

Hui-Bon-Hoa (2008). This non-standard mixing, modelled as a vertical effective turbulent diffusion applied on chemical elements (Chaboyer \& Zahn 1992, and Appendix A), is calibrated to reproduce the solar lithium abundance at the solar age in a solar model (we refer to Castro et al. 2016, for a detailed description of the calibration). The calibration is then used for the other models with different masses and metallicities. In this framework, it is important to point out that we focus here on the nonstandard mixing for chemicals (we refer to Appendix A for more details). This rotational mixing and the resulting lithium abundance strongly depend on internal transport mechanisms (e.g. Maeder 2009; Mathis 2013) and on angular momentum extraction by stellar winds (Skumanich 1972; Matt et al. 2015), as illustrated for example by the recent work by Amard et al. (2016). These processes are here calibrated as explained in Appendix A on the Sun and its rotational history through the effective vertical turbulent diffusion acting on chemicals. This may introduce a bias towards solar characteristics. However, this work constitutes a first step. In a near future, more realistic models will be computed where rotational and chemical evolutions will be treated coherently and simultaneously. These models will take all angular momentum processes and potentially different rotational histories into account.

The setup of the input physics used in our models is compatible with the setup used in the AMP models. Our models are calculated from the zero-age main sequence (ZAMS) to the helium burning in the core and for masses from 1.0 to $1.15 M_{\odot}$ with a step size of $0.05 M_{\odot}$. The main grid was calculated with a metallicity of $[\mathrm{Fe} / \mathrm{H}]=+0.20$. An additional model of the Lievolution was calculated for a $1.0 M_{\odot}$ star with $[\mathrm{Fe} / \mathrm{H}]=-0.20$. For comparison, the evolutionary track of the solar model computed by do Nascimento et al. (2009) is shown in Fig. 6.

We note that the grid of models contains only representative models chosen for an average mass and metallicity. The step size of $0.05 M_{\odot}$ is roughly the averaged uncertainty $(7 \%)$ of the detailed modelling approach found by Lebreton \& Goupil (2014), 
accounting for the observational uncertainties and those of the model approaches. The authors also found a typical uncertainty of the seismic age estimate of $\sim 10 \%$. Therefore this comparison provides a qualitative idea of whether the models and the measurements agree in general, but these model tracks are not calibrated to resemble specific stars on a case-to-case basis.

By focusing on stars for which detailed modelling has been performed and which were found to be without a companion, our sample is narrowed down to four stars. These are KIC $3656476($ age $=8.9 \mathrm{Gyr},[\mathrm{Fe} / \mathrm{H}]=+0.25 \mathrm{dex})$, KIC 6116048 (6.1 Gyr, $-0.18 \mathrm{dex})$, KIC 7680114 (6.9 Gyr, +0.15 dex), and KIC 10644253 (0.9 Gyr, +0.1 dex). The measured lithium abundances are compared against the stellar age from AMP-modelling for these four stars in Fig. 6. Here, the three stars KIC 10644253, KIC 7680114 and KIC 3656476 are of particular interest because they form a sequence of constant mass of $1.1 M_{\odot}$ (within their uncertainties) and a metallicity above the solar value, which spans stellar ages of between 1 and 9 Gyr. With its $1.05 \pm$ $0.03 M_{\odot}$, KIC 6116048 is closer to the solar mass but has a clearly sub-solar metallicity. In Fig. 6 the observed $A(\mathrm{Li})$ is plotted as a function of the estimated age from asteroseismology and compared to the predictions of these quantities from the abovedescribed model tracks.

\subsubsection{KIC 10644253}

The comparison depicted in Fig. 6 shows that the best agreement between measurements and models for age and lithium is found for KIC 10644253. The measured activity levels as well as the short rotation period reported by Salabert et al. (2016a,b) further confirm that this is a young stellar object.

\subsection{2. $\mathrm{KIC} 7680114$}

KIC 7680114 complies reasonably well with the evolutionary track of a star of $1.1 M_{\odot}$ with a metallicity of +0.2 dex. The star has a rotation period of 26.3 days, compatible with the solar period. The asteroseismic modelling places it at $\sim 7 \mathrm{Gyr}$, about $2.5 \mathrm{Gyr}$ older than the Sun. van Saders et al. (2016) showed that when mass or temperature are increased, the reduced efficiency of magnetic breaking starts earlier, which may explain this discrepancy.

\subsubsection{KIC 3656476}

The slowly rotating star KIC 3656476 is confirmed from stellar modelling and the comparison with the lithium abundance to be an old object. Although the $1.1 M_{\odot}$ evolutionary track of the TGEC modelling does not reach the age predicted by the AMP model, this is not a worrying disagreement. Taking the typical uncertainty of $\sim 7 \%$ on the seismic mass estimate, we find a good agreement with the Li evolution for a $1.05 M_{\odot}$ star. This uncertainty corresponds to $2 \sigma$ of the mass uncertainty of the stellar model reported by Creevey et al. (2017).

\subsubsection{KIC 6116048}

For KIC 6116048, the rotation period of $\sim 10$ days shorter than the value of the Sun suggests that this is a young star (García et al. 2014). On the other hand, this star has one of the lowest activities in our sample (Salabert et al. 2016a), and the seismic modelling suggests an age of $6 \mathrm{Gyr}$ (Creevey et al. 2017). In principle, it is possible that the rotation period from the literature could be half of the actual value (García et al. 2014). In this case, however, this star would fall out of the observed relation between $P_{\text {rot }}$ and A(Li.)

Despite the non-agreement of the age indicators, we find a general good agreement between $A(\mathrm{Li})$ measurement and the models. Within the conservative view on the uncertainty of the mass, the measured $A(\mathrm{Li})$ agrees well with the theoretical Li evolution for a 1.0 and $1.05 M_{\odot}$ star. Because $A(\mathrm{Li})$ remains relatively constant over a broad range in time, $\mathrm{Li}$ cannot be used to distinguish between the two scenarios. KIC 6116048 is a puzzling case that clearly needs further investigation.

\subsection{Results}

From the comparison of the observations with models, calculated for the determined seismic mass, we find $A(\mathrm{Li})$ in good agreement for all four single stars with available AMP modelling. For the Sun it has been shown that gravity waves have to be included in order to reproduce the solar rotation and lithium profile (Charbonnel \& Talon 2005). Although gravity waves were not explicitly included in the applied macroscopic hydrodynamical mixing modelling, they were implicitly taken into account through the calibration of the models to the Sun. The good agreement of measured and modelled $A(\mathrm{Li})$ in Fig. 6 shows that the four stars share the same internal physics as is working in the Sun.

\section{Conclusions}

We have presented the combined analysis of seismic, photometric, and spectroscopic values for a set of 18 solar-analogue stars. The sample is important and unique because not only the lithium abundance and the rotation period are known, but also the mass, radius, and age estimates for all stars from seismology. The rotation periods and seismic parameters used in this study were determined by several earlier studies (Mathur et al. 2012; García et al. 2014; Chaplin et al. 2014; Metcalfe et al. 2014; Creevey et al. 2017; Garcia et al., in prep.). For an overview of the literature values and references we refer to Table 1 . In a dedicated observing campaign we obtained high-resolution spectroscopy with the HERMES échelle spectrograph, allowing us to determine a consistent set of spectroscopic fundamental parameters, including the Li surface abundance. Based on our spectroscopic observations, we detected six new binary systems.

The surface abundance of lithium of a star is very sensitive to its rotation rate, mass, and metallicity. Masses from asteroseismology allowed us to select our targets accurately on the criterion of mass. Choosing a sample of stars within the very narrow mass range that is accepted as a solar-analogue enabled us to study the interplay of these parameters. From the sample of single stars, we could quantify a linear relationship between the rotation period and the $A(\mathrm{Li})$ for rotation periods shorter than solar. Binary stars show a larger scatter in the parameter plane. We demonstrated that observational restrictions can be overcome by calculating the actual rotational velocity $v_{\text {rot }}$ using the asteroseismically determined radius of the star. This allows a better comparison with model predictions.

By focusing on four single stars with available masses and ages reliably determined from detailed modelling with the AMPcode and comparing them with TGEC evolutionary track for the lithium abundance, we confirm the high degree of sensitivity of $A(\mathrm{Li})$ to the combination of stellar mass, metallicity, rotation rate, and age. For two of the "massive" solar analogues $\left(\sim 1.1 M_{\odot}\right)$ with detailed modelling, KIC 10644253 and 
KIC 7680114, the measured $A(\mathrm{Li})$ and the stellar mass and age from asteroseismology agree well with the predicted Li abundance. For the third "massive" solar analogue, KIC 3656476, a good agreement is found within the conservative mass uncertainty of $\sim 7 \%$. A similar case is the solar analogue with sub-solar metallicity, KIC 6116048. For this star we also find a good agreement with the modelled evolution of $A(\mathrm{Li})$ within the conservative mass uncertainty. The measured value of $A(\mathrm{Li})$ agrees with the plateau value found for $1.0 M_{\odot}$ star, but the rotation period indicates a young object, while seismology suggest a target that is older than the Sun. In principle, the rotation period could be underestimated by a factor of two, which would lead to a strong outlier in the $P_{\text {rot }}-A(\mathrm{Li})$ relation as depicted in Fig. 5, however.

The comparison of $A(\mathrm{Li})$ with age and the rotation rate demonstrates that gyrochronology is valid for stars younger than the Sun and until the age of the Sun. The small number of stars with individual frequency modelling does not allow us to draw further conclusions on their evolution with age. A larger data set is required to confirm the conclusions outlined here.

For these genuine solar analogues, a good agreement within the uncertainties is found between three independent approaches: the observed $A(\mathrm{Li})$ from spectroscopy, the stellar age and mass from asteroseismology, and the stellar model prediction of $A(\mathrm{Li})$ for representative TGEC-models. Because the TGEC-models for $A(\mathrm{Li})$ were calibrated to reproduce solar internal mixing, this consensus with the measured $A(\mathrm{Li})$ in the solar analogues may indicate that the solar analogues share the same internal mixing as the Sun. In this light, the solar value of $A(\mathrm{Li})$ is absolutely normal.

Acknowledgements. We thank the referee for a constructive report that allowed us to improve the article. We acknowledge the work of the team behind Kepler and Mercator. P.G.B. and R.A.G. acknowledge the ANR (Agence Nationale de la Recherche, France) program IDEE (No. ANR-12-BS05-0008) "Interaction Des Étoiles et des Exoplanètes". P.G.B. and R.A.G. also received funding from the CNES grants at CEA. J.D.N., M.C., and T.D. acknowledge the CNPq and the PPGF/UFRN. P.G.B. acknowledges the PPGF/UFRN for founding partially a scientific visit to the G3 team at UFRN, Natal, Brazil. D.S. and R.A.G. acknowledge the financial support from the CNES GOLF and PLATO grants. D.M. acknowledges financial support from the Spanish Ministerio de Economía y Competitividad under grant AYA2014-54348- C3-3-R. StM acknowledges support by the ERC through ERC SPIRE grant No. 647383. A.T. received funding from the European Research Council (ERC) under the European Union's Horizon 2020 research and innovation programme (grant agreement No. 670519: MAMSIE). SaM would like to acknowledge support from NASA grants NNX12AE17G and NNX15AF13G and NSF grant AST-1411685. The research leading to these results has received funding from the European Community's Seventh Framework Programme ([FP7/2007-2013]) under grant agreement No. 312844 (SPACEINN) and under grant agreement No. 269194 (IRSES/ASK). The observations are based on spectroscopy made with the Mercator Telescope, operated on the island of La Palma by the Flemish Community, at the Spanish Observatorio del Roque de los Muchachos of the Instituto de Astrofísica de Canarias. This research has made use of the SIMBAD database, operated at CDS, Strasbourg, France.

\section{References}

Aerts, C., Christensen-Dalsgaard, J., \& Kurtz, D. W. 2010, Asteroseismology (Springer Science+Business Media B.V.)

Alexander, D. R., \& Ferguson, J. W. 1994, ApJ, 437, 879

Allende Prieto, C., Beers, T. C., Wilhelm, R., et al. 2006, ApJ, 636, 804

Amard, L., Palacios, A., Charbonnel, C., Gallet, F., \& Bouvier, J. 2016, A\&A, 587, A105

Angulo, C., Arnould, M., Rayet, M., et al. 1999, Nucl. Phys. A, 656, 3

Asplund, M., Grevesse, N., Sauval, A. J., \& Scott, P. 2009, ARA\&A, 47, 481

Barklem, P. S. 2007, A\&A, 466, 327

Barklem, P. S., \& O’Mara, B. J. 2001, J. Phys. B At. Mol. Phys., 34, 4785

Barnes, S. A. 2007, ApJ, 669, 1167

Basu, S., \& Antia, H. M. 1997, MNRAS, 287, 189

Baumann, P., Ramírez, I., Meléndez, J., Asplund, M., \& Lind, K. 2010, A\&A, 519, A87
Beck, P. G., Allende Prieto, C., Van Reeth, T., et al. 2016, A\&A, 589, A27 Bensby, T., Feltzing, S., \& Oey, M. S. 2014, A\&A, 562, A71

Böhm-Vitense, E. 1958, ZAp, 46, 108

Bouvier, J. 2008, A\&A, 489, L53

Bouvier, J., Lanzafame, A. C., Venuti, L., et al. 2016, A\&A, 590, A78

Brun, A. S., \& Browning, M. K. 2017, Living Reviews in Solar Physics, submitted

Brun, A. S., Turck-Chièze, S., \& Zahn, J. P. 1998, in Structure and Dynamics of the Interior of the Sun and Sun-like Stars SOHO 6/GONG 98 Workshop, 439

Brun, A. S., Turck-Chièze, S., \& Zahn, J. P. 1999, ApJ, 525, 1032

Brun, A. S., García, R. A., Houdek, G., Nandy, D., \& Pinsonneault, M. 2015, Space Sci. Rev., 196, 303

Bruntt, H., Basu, S., Smalley, B., et al. 2012, MNRAS, 423, 122

Carlos, M., Nissen, P. E., \& Meléndez, J. 2016, A\&A, 587, A100

Castelli, F., \& Kurucz, R. L. 2004, Proc. IAU Symp. 210, poster A20 [arXiv: astro-ph/0405087]

Castro, M., Vauclair, S., Richard, O., \& Santos, N. C. 2009, A\&A, 494, 663

Castro, M., Duarte, T., Pace, G., \& do Nascimento, Jr., J. D. 2016, A\&A, 590, A94

Cayrel de Strobel, G. 1996, A\&ARv, 7, 243

Chaboyer, B., \& Zahn, J.-P. 1992, A\&A, 253, 173

Chaplin, W. J., Kjeldsen, H., Christensen-Dalsgaard, J., et al. 2011, Science, 332, 213

Chaplin, W. J., Basu, S., Huber, D., et al. 2014, ApJS, 210, 1

Chapman, S. 1917, MNRAS, 77, 540

Charbonnel, C., \& do Nascimento, Jr., J. D. 1998, A\&A, 336, 915

Charbonnel, C., \& Talon, S. 2005, Science, 309, 2189

Charbonnel, C., Vauclair, S., Maeder, A., Meynet, G., \& Schaller, G. 1994, A\&A, 283, 155

Choi, H.-J., Soon, W., Donahue, R. A., Baliunas, S. L., \& Henry, G. W. 1995, PASP, 107, 744

Clarke, D., MacDonald, E. C., \& Owens, S. 2004, A\&A, 415, 677

Coker, C. T., Pinsonneault, M., \& Terndrup, D. M. 2016, ApJ, 833, 122

Creevey, O., Metcalfe, T. S., Schultheis, M., et al. 2017, A\&A, 601, A67

Datson, J., Flynn, C., \& Portinari, L. 2014, MNRAS, 439, 1028

Delgado Mena, E., Israelian, G., González Hernández, J. I., et al. 2014, A\&A, 562, A92

do Nascimento, Jr., J. D., Castro, M., Meléndez, J., et al. 2009, A\&A, 501, 687

do Nascimento, Jr., J. D., da Costa, J. S., \& de Medeiros, J. R. 2010, A\&A, 519, A101

dos Santos, L. A., Meléndez, J., do Nascimento, J.-D., et al. 2016, A\&A, 592, A 156

Eddington, A. S. 1916, MNRAS, 77, 16

Eddington, A. S. 1926, The Internal Constitution of the Stars (Cambridge: Cambridge University Press)

Ekström, S., Georgy, C., Eggenberger, P., et al. 2012, A\&A, 537, A146

Epstein, C. R., Johnson, J. A., Dong, S., et al. 2010, ApJ, 709, 447

Fuhrmann, K., Axer, M., \& Gehren, T. 1993, A\&A, 271, 451

Gallet, F., \& Bouvier, J. 2013, A\&A, 556, A36

Gallet, F., \& Bouvier, J. 2015, A\&A, 577, A98

García, R. A., Ceillier, T., Salabert, D., et al. 2014, A\&A, 572, A34

GarciaLopez, R. J., \& Spruit, H. C. 1991, ApJ, 377, 268

Gehren, T. 1981, A\&A, 100, 97

Grevesse, N., \& Noels, A. 1993, in Perfectionnement de l'Association Vaudoise

des Chercheurs en Physique, eds. B. Hauck, S. Paltani, \& D. Raboud, 205

Gustafsson, B. 1998, Space Sci. Rev., 85, 419

Gustafsson, B. 2008, Phys. Scr. 130, 014036

Hui-Bon-Hoa, A. 2008, Ap\&SS, 316, 55

Iglesias, C. A., \& Rogers, F. J. 1996, ApJ, 464, 943

King, J. R., Krishnamurthi, A., \& Pinsonneault, M. H. 2000, AJ, 119, 859

Krishnamurthi, A., Terndrup, D. M., Pinsonneault, M. H., et al. 1998, ApJ, 493, 914

Lambert, D. L., \& Reddy, B. E. 2004, MNRAS, 349, 757

Lebreton, Y., \& Goupil, M. J. 2014, A\&A, 569, A21

Lehmann, H., Tkachenko, A., Semaan, T., et al. 2011, A\&A, 526, A124

Lind, K., Asplund, M., \& Barklem, P. S. 2009, A\&A, 503, 541

Ludwig, H.-G., Behara, N. T., Steffen, M., \& Bonifacio, P. 2009, A\&A, 502, L1

Maeder, A. 2009, Physics, Formation and Evolution of Rotating Stars (Berlin, Heidelberg: Springer)

Maeder, A., \& Zahn, J.-P. 1998, A\&A, 334, 1000

Mathis, S. 2013, in Lect. Notes Phys. 865, eds. M. Goupil, K. Belkacem, C. Neiner, F. Lignières, \& J. J. Green (Berlin: Springer Verlag), 23

Mathis, S., Palacios, A., \& Zahn, J.-P. 2004, A\&A, 425, 243

Mathur, S., Metcalfe, T. S., Woitaszek, M., et al. 2012, ApJ, 749, 152

Matt, S. P., Brun, A. S., Baraffe, I., Bouvier, J., \& Chabrier, G. 2015, ApJ, 799, L23

Meléndez, J., Ramírez, I., Casagrande, L., et al. 2010, Ap\&SS, 328, 193

Meléndez, J., Bergemann, M., Cohen, J. G., et al. 2012, A\&A, 543, A29 
Meléndez, J., Ramírez, I., Karakas, A. I., et al. 2014a, ApJ, 791, 14 Meléndez, J., Schirbel, L., Monroe, T. R., et al. 2014b, A\&A, 567, L3

Metcalfe, T. S., Creevey, O. L., \& Christensen-Dalsgaard, J. 2009, ApJ, 699, 373

Metcalfe, T. S., Creevey, O. L., Doğan, G., et al. 2014, ApJS, 214, 27

Monroe, T. R., Meléndez, J., Ramírez, I., et al. 2013, ApJ, 774, L32

Pace, G., Castro, M., Meléndez, J., Théado, S., \& do Nascimento, Jr., J.-D. 2012, A\&A, 541, A150

Paquette, C., Pelletier, C., Fontaine, G., \& Michaud, G. 1986, ApJS, 61, 177

Paxton, B., Cantiello, M., Arras, P., et al. 2013, ApJS, 208, 4

Pinsonneault, M. H. 2010, in IAU Symp., 268, eds. C. Charbonnel, M. Tosi, F. Primas, \& C. Chiappini, 375

Ramírez, I., Meléndez, J., Bean, J., et al. 2014, A\&A, 572, A48

Raskin, G. 2011, Ph.D. Thesis, Institute of Astronomy, Katholieke Universiteit Leuven, Belgium

Raskin, G., van Winckel, H., Hensberge, H., et al. 2011, A\&A, 526, A69

Rebolo, R., \& Beckman, J. E. 1988, A\&A, 201, 267

Reddy, B. E., Tomkin, J., Lambert, D. L., \& Allende Prieto, C. 2003, MNRAS, 340,304

Rieutord, M. 2006, A\&A, 451, 1025

Robles, J. A., Lineweaver, C. H., Grether, D., et al. 2008, ApJ, 684, 691

Rogers, F. J., \& Nayfonov, A. 2002, ApJ, 576, 1064

Salabert, D., García, R. A., Beck, P. G., et al. 2016a, A\&A, 596, A31

Salabert, D., Régulo, C., García, R. A., et al. 2016b, A\&A, 589, A118

Schatzman, E. 1996, J. Fluid Mech., 322, 355

Shulyak, D., Tsymbal, V., Ryabchikova, T., Stütz, C., \& Weiss, W. W. 2004 A\&A, 428, 993

Skumanich, A. 1972, ApJ, 171, 565

Sneden, C. A. 1973, Ph.D. Thesis, The Univerisity of Texas at Austin, USA

Somers, G., \& Pinsonneault, M. H. 2016, ApJ, 829, 32

Sousa, S. G., Santos, N. C., Israelian, G., Mayor, M., \& Monteiro, M. J. P. F. G. 2007, A\&A, 469, 783

Spiegel, E. A., \& Zahn, J. P. 1992, A\&A, 265, 106

Sweet, P. A. 1950, MNRAS, 110, 548

Takeda, Y., Honda, S., Kawanomoto, S., Ando, H., \& Sakurai, T. 2010, A\&A, 515, A93

Talon, S., \& Charbonnel, C. 1998, A\&A, 335, 959

Talon, S., \& Charbonnel, C. 2005, A\&A, 440, 981

Théado, S., \& Vauclair, S. 2003, ApJ, 587, 777

Thompson, M. J., Toomre, J., Anderson, E. R., et al. 1996, Science, 272, 1300

Tkachenko, A. 2015, A\&A, 581, A129

Tkachenko, A., Lehmann, H., Smalley, B., Debosscher, J., \& Aerts, C. 2012, MNRAS, 422, 2960

Tsymbal, V. 1996, in M.A.S.S., Model Atmospheres and Spectrum Synthesis, eds. S. J. Adelman, F. Kupka, \& W. W. Weiss, ASP Conf. Ser., 108, 198 van den Heuvel, E. P. J., \& Conti, P. S. 1971, Science, 171, 895

van Saders, J. L., Ceillier, T., Metcalfe, T. S., et al. 2016, Nature, 529, 181

Zahn, J.-P. 1992, A\&A, 265, 115

Zahn, J.-P. 1994, A\&A, 288, 829

\section{Appendix A: Physics of the TGEC grid}

A grid of models was calculated with the ToulouseGeneva stellar evolution code (TGEC, Hui-Bon-Hoa 2008; do Nascimento et al. 2009). The initial chemical mixture relative to the hydrogen content was chosen as the solar mixture from Grevesse \& Noels (1993). The equations of state are derived from the OPAL tables (Rogers \& Nayfonov 2002), and we used the OPAL96 opacity tables (Iglesias \& Rogers 1996) completed by the Alexander \& Ferguson (1994) low-temperature opacities. For the nuclear reaction rates, we used the NACRE compilation (Angulo et al. 1999) with the Bahcall screening routine.
Convection was modelled according to the Böhm-Vitense (1958) formalism of the mixing-length theory. The mixinglength parameter $\alpha=l / H_{p}=1.69$, where $l$ is the characteristic mixing length and $H_{p}$ is the pressure scale height, is a free parameter that calibrates the radius of a solar model. Below the convective zone, we introduced convective undershooting with a depth of $0.09 \mathrm{H}_{p}$ so that in a solar model the combined mixing reaches the depth deduced by helioseismology $\left(r_{c z} / R_{\odot}=0.713 \pm 0.001\right.$; Basu \& Antia 1997). In the radiative zone, microscopic diffusion, which is the process of element segregation by gravitational and thermal diffusion (Eddington 1916; Chapman 1917), was treated by using the Paquette et al. (1986) method for collisions between charged ions with a screened Coulomb potential. For a complete description of the microphysics used by these models, we refer to Théado \& Vauclair (2003), do Nascimento et al. (2009), Castro et al. (2016).

The meridional circulation in stellar radiation zones (e.g. Eddington 1926; Sweet 1950), which is driven by the internal stresses induced by rotation (e.g. Zahn 1992; Rieutord 2006; Mathis 2013), was modelled as prescribed by Zahn (1992). Zahn (1992) demonstrated that these meridional flows transport angular momentum, creating shears that become unstable with a stronger turbulent transport in the horizontal than in the vertical direction because of the stable stratification. As demonstrated by Chaboyer \& Zahn (1992), the vertical advection of chemicals by the meridional circulation is transformed into an effective vertical diffusion by the strong horizontal turbulence. Similarly, the transition layer at the bottom of the convection zone between its latitudinal differential rotation and the solidbody rotation of the radiative core (the so-called tachocline) undergoes the same strong anisotropic turbulence (with a much stronger turbulent transport in the horizontal than in the vertical direction). The resulting turbulent transport reduces the differential rotation and inhibits its spread deep inside the radiative interior (Spiegel \& Zahn 1992). Brun et al. (1998) showed that the vertical turbulent transport of chemicals in these layers can be modelled by an exponential diffusion coefficient, which is added to the previously described effective vertical turbulent diffusion. The resulting total vertical turbulent diffusion coefficient in the transport equation for the mean concentration of the different chemical species was here calibrated to reproduce the solar lithium abundance in a solar model as in Castro et al. (2016). In our models, we solved only the transport equation for chemicals. The angular momentum history was not computed explicitly since the equation for the transport of angular momentum is not solved in our models, but it is implicitly taken into account through calibration constants of the effective vertical diffusion, which are implicit functions of rotation and shear (Chaboyer \& Zahn 1992; Zahn 1992; Hui-Bon-Hoa 2008; Castro et al. 2016). 